\begin{tabular}{|c|l|}
\hline Title & Persistent chimera states in nonlocally coupled phase oscillators \\
\hline Author(s) & Suda, Y usuke; Okuda, Koji \\
\hline Citation & $\begin{array}{l}\text { Physical review E, 92(6), 60901 } \\
\text { https://doi.org/10.1103/PhysRevE.92.060901 }\end{array}$ \\
\hline Issue Date & 2015-12-14 \\
\hline Doc URL & http://hdl.handle.net/2115/60634 \\
\hline Type & article \\
\hline File Information & PhysRevE.92.060901.pdf \\
\hline
\end{tabular}

Instructions for use 


\title{
Persistent chimera states in nonlocally coupled phase oscillators
}

\author{
Yusuke Suda and Koji Okuda \\ Division of Physics, Hokkaido University, Sapporo 060-0810, Japan
}

(Received 13 August 2015; published 14 December 2015)

\begin{abstract}
Chimera states in the systems of nonlocally coupled phase oscillators are considered stable in the continuous limit of spatially distributed oscillators. However, it is reported that in the numerical simulations without taking such limit, chimera states are chaotic transient and finally collapse into the completely synchronous solution. In this Rapid Communication, we numerically study chimera states by using the coupling function different from the previous studies and obtain the result that chimera states can be stable even without taking the continuous limit, which we call the persistent chimera state.
\end{abstract}

DOI: 10.1103/PhysRevE.92.060901

PACS number(s): 05.45.Xt, 89.75.Kd

The behavior of coupled oscillator systems can describe various pattern formations in a wide range of scientific fields $[1,2]$. In the systems of nonlocally coupled identical oscillators, there often appears a strange phenomenon called the chimera state, which is characterized by the coexistence of coherent and incoherent domains, where the former domain consists of phase-locked oscillators and the latter domain consists of drifting oscillators with spatially changing frequencies [3-26]. This interesting phenomenon was first discovered in the system of nonlocally coupled phase oscillators obeying the evolution equation

$$
\frac{\partial}{\partial t} \theta(x, t)=\omega-\int d x^{\prime} G\left(x-x^{\prime}\right) \Gamma\left(\theta(x, t)-\theta\left(x^{\prime}, t\right)\right)
$$

with $2 \pi$-periodic phases $\theta(x)$ on a finite interval $x \in[0,1]$ under the periodic boundary condition, a smooth $2 \pi$-periodic coupling function $\Gamma$, and the kernel $G(y)=(\kappa / 2) \exp (-\kappa|y|)$, where a constant $1 / \kappa$ denotes the coupling range [3]. Recently, similar spatiotemporal patterns have been found in various systems using, e.g., the logistic maps [14,15], Rössler systems [15], and FitzHugh-Nagumo oscillators [18].

In the study of the chimera state, the system, Eq. (1), with the sine coupling [27]

$$
\Gamma(\phi)=-\sin (\phi+\alpha)
$$

is particularly important because of its simplicity and generality. In fact, this coupling function was used also in the first discovery of the chimera state [3]. For numerical simulations, we usually discretize Eq. (1) into such form as Eq. (3). In the simulations of such discretized systems, we can confirm that chimera states are surely stable in the continuous limit $N \rightarrow \infty$. However, the stability of chimera states in finitely discretized systems is questioned. In fact, it is reported that when $N$ is finite, chimera states with the sine coupling are chaotic transient and finally collapse into the completely synchronous solution [12,13,26].

Recently, Ashwin and Burylko proposed the weak chimera similar to the chimera state, which is defined by the coexistence of frequency-synchronous and -asynchronous oscillators in the systems of coupled indistinguishable phase oscillators but is not necessarily spatially structured as coherent and incoherent domains [28]. They studied the weak chimera in some types of networks composed of the minimal number of oscillators with the Hansel-Mato-Meunier coupling, Eq. (4), and demonstrated that the weak chimera can be persistent (nontransient). In this
Rapid Communication, we study chimera states in the systems of nonlocally coupled phase oscillators with the Hansel-MatoMeunier coupling by numerical simulation, and demonstrate that it is possible for persistent chimera states to appear.

As a model, we consider a ring of $N$ identical nonlocally coupled phase oscillators described as

$$
\dot{\theta}_{j}(t)=\omega+\frac{1}{2 R} \sum_{k=j-R}^{j+R} \Gamma\left(\theta_{j}(t)-\theta_{k}(t)\right)
$$

with $2 \pi$-periodic phases $\theta_{j}(j=1, \ldots, N)$. This model corresponds to a spatially discretized version of Eq. (1) with a constant kernel within a certain range. The natural frequency $\omega$ of the oscillators can be set to zero without loss of generality, and the nonlocal coupling range $R$ needs to satisfy $1<R<(N-1) / 2$. In this Rapid Communication, we fix $R / N \sim 0.35$. As the coupling function $\Gamma(\phi)$, we choose the Hansel-Mato-Meunier coupling [29]

$$
\Gamma(\phi)=-\sin (\phi+\alpha)+r \sin (2 \phi)
$$

where $\alpha$ is the phase lag parameter of the fundamental harmonic component and $r$ is the amplitude ratio of the second harmonic component. For $r=0$, Eq. (4) recovers the sine coupling, Eq. (2). In the systems of globally coupled phase oscillators, it is known that such higher harmonic components in the coupling function are responsible for a rich variety of synchronous patterns excluded by the sine coupling [29-34]. Therefore we expect that also in the systems of nonlocally coupled phase oscillators with Eq. (4), we could observe new chimera patterns excluded by the sine coupling.

First, we consider the case of sufficiently large $N$ corresponding to the continuous limit. Figure 1 shows the results of numerical simulation of Eq. (3) with Eq. (4) for several $r \geqslant 0$. For all the simulations of the present Rapid Communication, we used the fourth-order Runge-Kutta method with time interval $\Delta t=0.01$. In Fig. 1 , we fix $\alpha=1.46$, for which chimera states are observed in the case of the sine coupling $(r=0)$. As initial conditions, we used

$$
\theta_{j}(0)=6 \exp \left[-30\left(\frac{j}{N}-\frac{1}{2}\right)^{2}\right] R_{j},
$$

where $R_{j} \in[-1 / 2,1 / 2]$ is a uniform random number, which is so close to a chimera state as to assist its emergence [6]. 

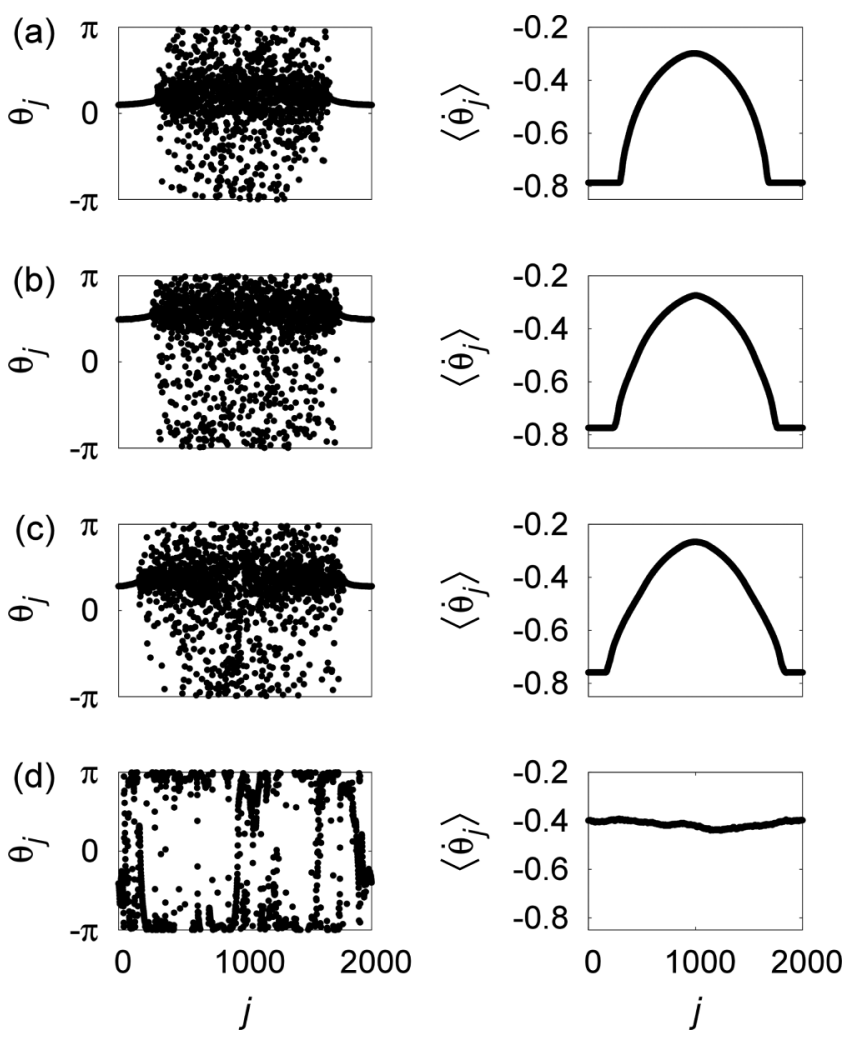

FIG. 1. Results of numerical simulation of Eq. (3) with $N=2000$ and $\alpha=1.46$. In each row, the left panel shows the snapshot of phase $\theta_{j}$, and the right panel shows the profile of the average frequency $\left\langle\dot{\theta}_{j}\right\rangle$ with $T=5000$ and $t_{\text {rel }}=2000$ in Eq. (6). For (a) $r=0.001$, (b) $r=0.03$, and (c) $r=0.06$, chimera states are observed, while they are not observed for (d) $r=0.12$.

In our simulation, chimera states are observed for $r<0.073$ as shown in Figs. 1(a)-1(c). The phase pattern (left panels) is clearly separated into coherent and incoherent domains, which is characteristic of the chimera state. From the right panels of this figure, we can see that the average frequency

$$
\left\langle\dot{\theta}_{j}\right\rangle(T)=\frac{1}{T} \int_{t_{\mathrm{rel}}}^{t_{\mathrm{rel}}+T} \dot{\theta}_{j}(t) d t
$$

of each oscillator in the coherent domain is almost constant, where $T$ is the measurement time and $t_{\mathrm{rel}}$ is the relaxation time, while the frequency in the incoherent domain varies continuously. For $r \geqslant 0.073$, chimera states gradually disappear as $r$ increases. In addition, for $r \geqslant 0.110$, chimera states are not observed, but each oscillator evolves almost independently, where the average frequency seems to converge to a constant value in the limit of $T \rightarrow \infty$, though the frequency in Fig. 1(d) still exhibits some fluctuations due to a finite $T$. The survey of these behaviors is depicted in Fig. 2.

From the linear stability analysis, it is found that the completely synchronous solution $\theta_{1}=\theta_{2}=\cdots=\theta_{N}$ to Eq. (3) with Eq. (4) is stable for $r<(\cos \alpha) / 2(\simeq 0.055$ at $\alpha=1.46)$. Moreover, chimera states also appear to be stable in this parameter region [see Figs. 1(a) and 1(b)]. However, it is reported that when $N$ is finite, chimera states at $r=0$ are transient and finally collapse into the completely synchronous

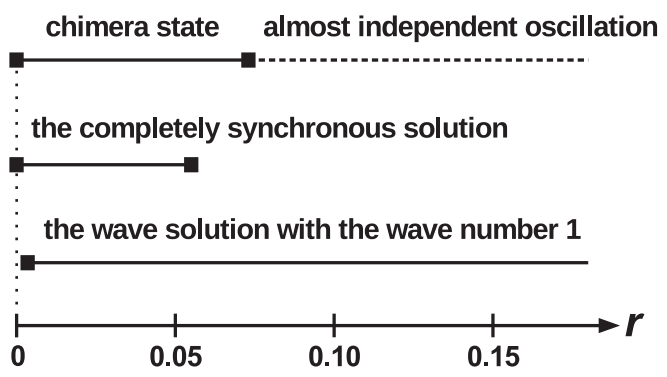

FIG. 2. Phase diagram of stable solutions to Eq. (3) with Eq. (4) at $\alpha=1.46$ in the continuous limit $(N=2000)$. Horizontal lines denote the stability regions of each solution. We have not clearly determined the transition between the chimera state and almost independent oscillation yet. Note that the stability region of the wave solution with the wave number $k=1$ does not cover $r=0$.

state [13]. We below confirm whether these chimera states, particularly for $r>0$, are transient or really stable even when $N$ is finite.

Figure 3 shows the average lifetime $\tau$ of the chimera state for $N=30$, as increasing $r$ from 0 to $(\cos \alpha) / 2 \simeq 0.055$. Here we regard the lifetime of the chimera state as the time at which the completely synchronous state appears, i.e., the global order parameter

$$
Z(t)=\left|\frac{1}{N} \sum_{k=1}^{N} e^{i \theta_{k}}\right|
$$

reaches $Z(t)=1$. As for the chimera state in the finite $N$ cases, it should be noted that it is difficult to judge the emergence of the chimera state, because the spatial position of the chimera state does not stay still but fluctuates [11], in particular, more violently as $N$ becomes smaller. In fact, in the case of $N=30$, we could not observe the characteristic profile of the average frequency as in the right panels of Figs. 1(a)-1(c). However, we observed that the coherent domain exists in the phase snapshots

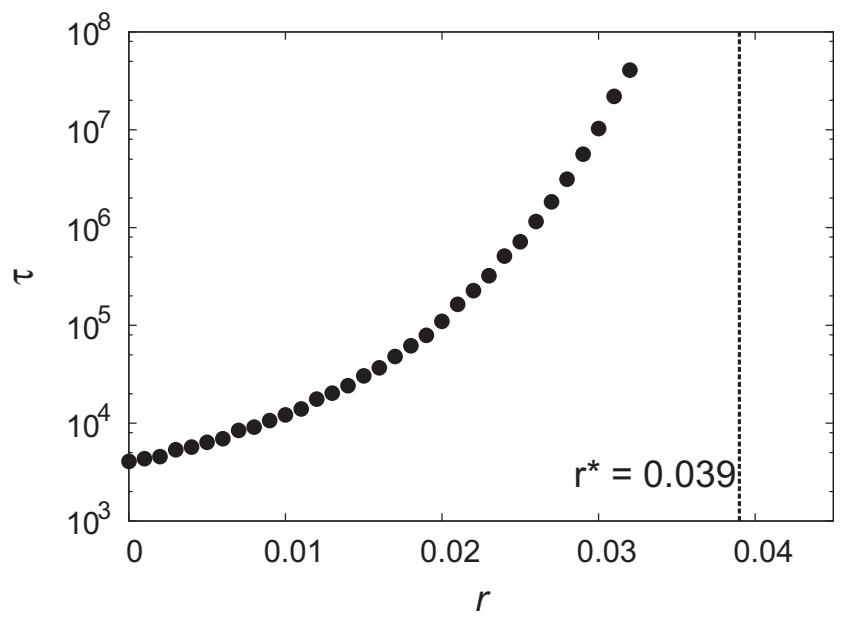

FIG. 3. Average lifetime $\tau$ of the chimera state as varying $r$ at the parameters $N=30$ and $\alpha=1.46$. A point in the figure is the average over 1000 simulations from different initial conditions obeying Eq. (5). 


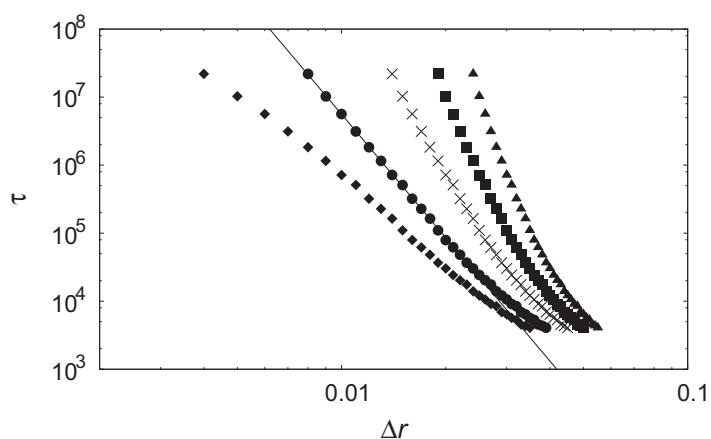

FIG. 4. Log-log plot of the data $(\Delta r, \tau)$ for $r^{*}=0.035$ (diamond), 0.039 (circle), 0.045 (cross), 0.050 (square), and 0.055 (triangle). The data for $r^{*}=0.039$ are fitted linearly by the least-squares method (black line), where we used only the data $\tau \geqslant 300000$ to obtain better linearity for this fitting.

as in the left panels of Figs. 1(a)-1(c), which convinces us of the emergence of the chimera state.

For Fig. 3, it should be noted that there is a possibility that chimera states collapse into a stable solution other than the completely synchronous state. From the linear stability analysis, we can show that the wave solution $\theta_{i}=\theta_{1}+$ $2 \pi k(i-1) / N[23,25]$ with the wave number $k=1$ is also stable for $r \geqslant 0.003$ (Fig. 2). This implies that chimera states may collapse into the wave solution. However, we never observed such collapse in our simulations from 1000 different initial conditions, Eq. (5), at each $r$.

As $r$ is increased, the average lifetime $\tau$ increases monotonically, and appears to diverge to infinity at a certain $r=r^{*}$. Assuming some values as $r^{*}$, we obtain Fig. 4 by the log-log plot of the data $(\Delta r, \tau)$, where $\Delta r \equiv r^{*}-r$. From this figure, we can assume the power law

$$
\tau \propto(\Delta r)^{-\zeta}
$$

and we determine $r^{*} \simeq 0.039$ from the best linear fitting of the data. Since $r^{*}<(\cos \alpha) / 2 \simeq 0.055$, this implies that there exists a parameter region where the chimera state (with infinite lifetime) and the completely synchronous state are bistable even in the finite $N$ cases. However, we cannot exclude the possibility of $r^{*}=(\cos \alpha) / 2$, because it is difficult to obtain the exact value of $r^{*}$ due to divergent simulation time.

Next, we investigate the chimera state of $N=30$ for $r>(\cos \alpha) / 2$, where the completely synchronous state is unstable. The possibility that chimera states appear in the region without the stable completely synchronous state differs from the case of the sine coupling. In this region, chimera states cannot collapse into the completely synchronous state. Though the wave solution with $k=1$ is stable in this region, we never observed that chimera states collapse into the wave solution within our maximum simulation time $t=2 \times 10^{8}$. Therefore, the collapse of the chimera state should not occur if other stable nonchimera solutions do not exist. Though we searched for stable nonchimera solutions other than the wave solution by extensive numerical simulations, we could not find any such solutions. From the above results, we conclude that, in a certain range of $r>(\cos \alpha) / 2$, the chimera state and the wave solution are bistable, and the chimera state can be

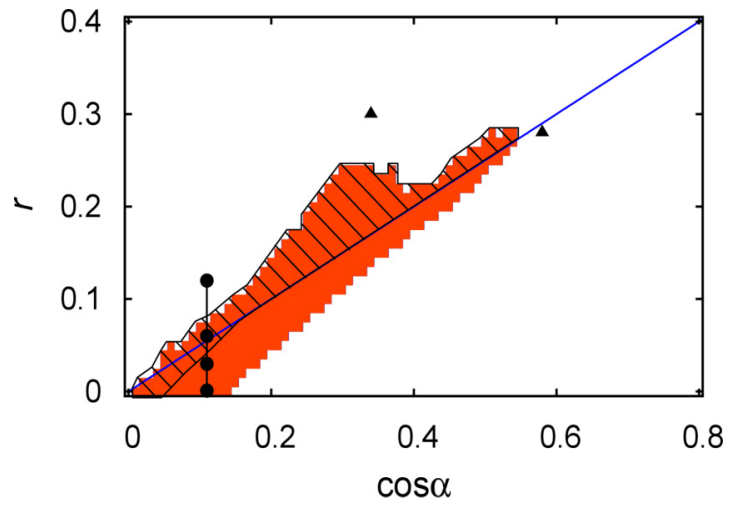

FIG. 5. (Color online) Stability region of the chimera state (red) on the $(\cos \alpha, r)$ plane in the continuous limit $(N=2000)$. The hatched region corresponds to the persistent chimera state, that is, the stability region of the chimera state in the case of $N=30$. The blue line denotes $r=(\cos \alpha) / 2$, and the completely synchronous solution is stable for $r<(\cos \alpha) / 2$. Black circles denote the parameter values of Fig. 1. Black triangles denote the parameter values of Fig. 7, where multichimera states appear.

persistent (nontransient) even in the finite $N$ cases. This result is consistent with $\tau \rightarrow \infty$ for $r>r^{*}$, as seen in Fig. 3 .

Investigating the chimera states in the $(\cos \alpha, r)$ parameter space, we obtained Fig. 5. The red region corresponding to the chimera state in the continuous limit $(N=2000)$ is spread around $r=(\cos \alpha) / 2$. In the finite $N$ cases, the chimera state for small $r$ becomes transient, while the chimera state for large $r$ remains persistent, as seen at least for $r>(\cos \alpha) / 2$ of the red region. For $\cos \alpha<0.15$, we can see that there exists a region $r^{*}<r<(\cos \alpha) / 2$ where the chimera state is persistent in the case of $N=30$. Note that the stability region of the persistent chimera state (hatched in Fig. 5) extends to the $r=0$ line, which implies that the chimera state with the sine coupling can be persistent (nontransient) even in the finite $N$ cases. Specifically, the average lifetime $\tau$ of the chimera state increases similarly to Fig. 3 as $\cos \alpha$ is decreased on the $r=0$ line, and diverge at $\cos \alpha^{*} \simeq 0.044$. However, this fact does not contradict the previous study that shows the transient chimera state [13], because the parameter $\alpha$ in that study corresponds to the line of black circles in Fig. 5, which has a larger $\cos \alpha$ than our hatched region on the $r=0$ line.

In summary, we studied chimera states in the systems of nonlocally coupled phase oscillators, Eq. (3), with the HanselMato-Meunier coupling, Eq. (4), by numerical simulations, motivated by the result that chimera states with the sine coupling, Eq. (2), in finitely discretized systems are chaotic transient and finally collapse into the completely synchronous state [13]. The existence of chimera states was examined in the parameter space $(\alpha, r)$ in Eq. (4), and the chimera states were observed around $r=(\cos \alpha) / 2$ in the continuous limit $N \rightarrow \infty$. For $r<(\cos \alpha) / 2$, the chimera state and the completely synchronous state can be bistable. In this region of the finite $N$ cases, the chimera state is transient for $r<r^{*}$, but it is persistent for $r^{*}<r<(\cos \alpha) / 2$. Moreover, even for $r>(\cos \alpha) / 2$, it is persistent in the region where the chimera state in $N \rightarrow \infty$ is stable. At first, we expected the chimera state to become persistent due to the destabilization of the 

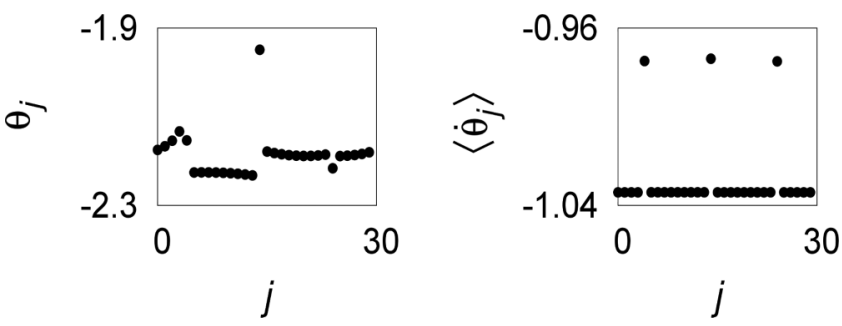

FIG. 6. Weak chimera for Eq. (3) with $N=30, \alpha=1.46$, and $r=0.032$, which are the parameter values on the line of black circles in Fig. 5. The snapshot of phase $\theta_{j}$ (left) and the profile of the average frequency $\left\langle\dot{\theta}_{j}\right\rangle$ (right).

completely synchronous state by the effect of $r$, but have obtained the persistent chimera state not only in the unstable region of the completely synchronous state as expected but also in its stable region. As a result, we have discovered that the chimera state in the case of the sine coupling can also be persistent by using appropriate $\alpha$ in the stability region of the completely synchronous state. Though we have numerically found the persistent chimera state in this Rapid Communication, its bifurcation-theoretical understanding is still an open problem.

When we investigated the collapse of chimera states at $\alpha=1.46$, we infrequently observed that a chimera state collapses into a weak chimera characterized by the coexistence of frequency-synchronous and -asynchronous oscillators [28,35], as shown in Fig. 6. In [28], the existence of weak chimeras for Eq. (3) with Eq. (4) is confirmed in the system with a small number of oscillators $(N=4,6$, and 10). In our numerical simulation with a larger number of oscillators $(N=30)$, such
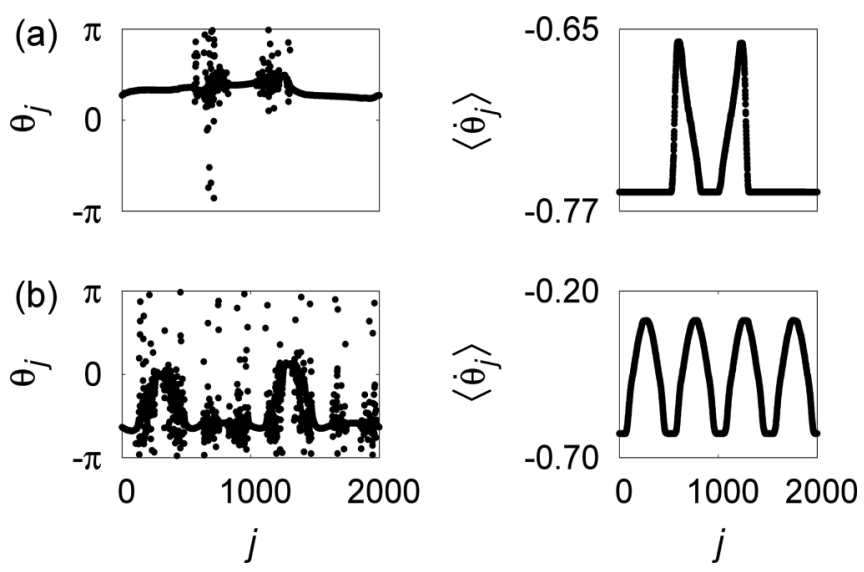

FIG. 7. Multichimera states for Eq. (3) with $N=2000$. The left panels show the snapshot of phase $\theta_{j}$, and the right panels show the profile of the average frequency $\left\langle\dot{\theta}_{j}\right\rangle$ with $T=5000$ and $t_{\text {rel }}=2000$ in Eq. (6). Parameter values are (a) $\alpha=0.95$ and $r=0.28$, and (b) $\alpha=1.22$ and $r=0.30$, which are plotted in Fig. 5 .

a weak chimera is stable in a small range of $r<(\cos \alpha) / 2$, for example, $0.032 \leqslant r \leqslant 0.040$ at $\alpha=1.46$.

Moreover, as other solutions, we observed multichimera states, which have two or more incoherent domains [18,23-25], for Eq. (3) with Eq. (4) in the continuous limit $(N=2000)$, as shown in Fig. 7. Other than the black triangles in Fig. 5, we observed multichimera states in a large region of the parameter space, though we do not describe the region in detail because it is beyond the scope of the present Rapid Communication.
[1] Y. Kuramoto, Chemical Oscillation, Waves, and Turbulence (Springer, Berlin, 1984).

[2] A. Pikovsky, M. Rosenblum, and J. Kurths, Synchronization: A Universal Concept in Nonlinear Sciences (Cambridge University Press, Cambridge, 2003).

[3] Y. Kuramoto and D. Battogtokh, Nonlinear Phenom. Complex Syst. 5, 380 (2002).

[4] D. M. Abrams and S. H. Strogatz, Phys. Rev. Lett. 93, 174102 (2004).

[5] S.-I. Shima and Y. Kuramoto, Phys. Rev. E 69, 036213 (2004).

[6] D. M. Abrams and S. H. Strogatz, Int. J. Bifurcation Chaos 16, 21 (2006).

[7] D. M. Abrams, R. Mirollo, S. H. Strogatz, and D. A. Wiley, Phys. Rev. Lett. 101, 084103 (2008).

[8] E. Ott and T. M. Antonsen, Chaos 18, 037113 (2008).

[9] E. Ott and T. M. Antonsen, Chaos 19, 023117 (2009).

[10] E. A. Martens, C. R. Laing, and S. H. Strogatz, Phys. Rev. Lett. 104, 044101 (2010).

[11] O. E. Omel'chenko, M. Wolfrum, and Y. L. Maistrenko, Phys. Rev. E 81, 065201(R) (2010).

[12] M. Wolfrum, O. E. Omel'chenko, S. Yanchuk, and Y. L. Maistrenko, Chaos 21, 013112 (2011).

[13] M. Wolfrum and O. E. Omel'chenko, Phys. Rev. E 84, 015201(R) (2011).
[14] I. Omelchenko, Y. Maistrenko, P. Hövel, and E. Schöll, Phys. Rev. Lett. 106, 234102 (2011).

[15] I. Omelchenko, B. Riemenschneider, P. Hövel, Y. Maistrenko, and E. Schöll, Phys. Rev. E 85, 026212 (2012).

[16] M. R. Tinsley, S. Nkomo, and K. Showalter, Nat. Phys. 8, 662 (2012).

[17] A. M. Hagerstrom, T. E. Murphy, R. Roy, P. Hövel, I. Omelchenko, and E. Schöll, Nat. Phys. 8, 658 (2012).

[18] I. Omelchenko, O. E. Omel'chenko, P. Hövel, and E. Schöll, Phys. Rev. Lett. 110, 224101 (2013).

[19] E. A. Martens, S. Thutupalli, A. Fourriére, and O. Hallatschek, Proc. Natl. Acad. Sci. USA 110, 10563 (2013).

[20] L. Schmidt, K. Schönleber, K. Krischer, and V. García-Morales, Chaos 24, 013102 (2014).

[21] Y. Zhu, Z. Zheng, and J. Yang, Phys. Rev. E 89, 022914 (2014).

[22] S. W. Haugland, L. Schmidt, and K. Krischer, Sci. Rep. 5, 9883 (2015).

[23] G. C. Sethia, A. Sen, and F. M. Atay, Phys. Rev. Lett. 100, 144102 (2008).

[24] Y. L. Maistrenko, A. Vasylenko, O. Sudakov, R. Levchenko, and V. L. Maistrenko, Int. J. Bifurcation Chaos 24, 1440014 (2014).

[25] J. Xie, E. Knobloch, and H.-C. Kao, Phys. Rev. E 90, 022919 (2014). 
[26] D. P. Rosin, D. Rontani, N. D. Haynes, E. Schöll, and D. J. Gauthier, Phys. Rev. E 90, 030902(R) (2014).

[27] H. Sakaguchi and Y. Kuramoto, Prog. Theor. Phys. 76, 576 (1986).

[28] P. Ashwin and O. Burylko, Chaos 25, 013106 (2015).

[29] D. Hansel, G. Mato, and C. Meunier, Phys. Rev. E 48, 3470 (1993).
[30] H. Daido, Prog. Theor. Phys. 88, 1213 (1992).

[31] K. Okuda, Physica D 63, 424 (1993).

[32] H. Daido, Physica D 91, 24 (1996).

[33] H. Kori and Y. Kuramoto, Phys. Rev. E 63, 046214 (2001).

[34] P. Ashwin, O. Burylko, and Y. Maistrenko, Physica D 237, 454 (2008).

[35] C. Bick and P. Ashwin, arXiv:1509.08824 (2015). 